\title{
Self-similar solutions of viscous and resistive ADAFs with thermal conduction
}

\author{
Kazem Faghei
}

\begin{abstract}
We have studied the effects of thermal conduction on the structure of viscous and resistive advection-dominated accretion flows (ADAFs). The importance of thermal conduction on hot accretion flow is confirmed by observations of hot gas that surrounds Sgr $\mathrm{A}^{*}$ and a few other nearby galactic nuclei. In this research, thermal conduction is studied by a saturated form of it, as is appropriated for weakly-collisional systems. It is assumed the viscosity and the magnetic diffusivity are due to turbulence and dissipation in the flow. The viscosity also is due to angular momentum transport. Here, the magnetic diffusivity and the kinematic viscosity are not constant and vary by position and $\alpha$-prescription is used for them. The govern equations on system have been solved by the steady selfsimilar method. The solutions show the radial velocity is highly subsonic and the rotational velocity behaves sub-Keplerian. The rotational velocity for a specific value of the thermal conduction coefficient becomes zero. This amount of conductivity strongly depends on magnetic pressure fraction, magnetic Prandtl number, and viscosity parameter. Comparison of energy transport by thermal conduction with the other energy mechanisms implies that thermal conduction can be a significant energy mechanism in resistive and magnetized ADAFs. This property is confirmed by non-ideal magnetohydrodynamics (MHD) simulations.
\end{abstract}

Keywords accretion, accretion discs - conduction magnetohydrodynamics: MHD

Kazem Faghei

School of Physics, Damghan University, Damghan, Iran e-mail: kfaghei@du.ac.ir

Received: 14 October 2011 / Accepted: 30 November 2011

\section{Introduction}

The observational features in active galactic nuclei (AGN) and X-ray binaries can be successfully explained by the standard geometrically thin, optically thick accretion disc model (Shakura \& Sunyaev 1973). The motion of the matter in the standard thin model of the accretion disc is approximately Keplerian, and the energy released in the accreting gas is radiated away locally. In the past two decades, another type of accretion flow has been considered that the energy released due to heating processes in the flow may be trapped within accreting gas. As, only the small fraction of the energy released in the accretion flow is radiated away due to inefficient cooling, and most of the energy is stored in the accretion flow and advected to the central object. This type of accretion flow is called as advection-dominated accretion flow (ADAF). The models of ADAF have been studied by a number of researchers (e.g. Ichimaru 1977; Rees et al. 1982; Narayan \& Yi 1994; Abramowicz et al. 1995; Blandford \& Begelman 1999; Ogilvie 1999).

The observations of black holes confirm existence of hot accretion flow that contrasted with classical cold and thin accretion disc model (Shakura \& Sunyaev 1973). Hot accretion flows can be seen in supermassive black holes of galactic nuclei and during quiescent of accretion onto stellar-mass black holes in X-ray transients (e.g., Lasota et al. 1996; Esin et al. 1997, 2001; Narayan et al. 1998a; Menou et al. 1999; Di Matteo et al. 2000; see Narayan et al. 1998b; Melia \& Falcke 2001; Narayan 2002; Narayan \& Quataert 2005 for reviews). Chandra observations provide constraints on the density and temperature of gas at or near the the Bondi capture radius in Sgr $\mathrm{A}^{*}$ and several nearby galactic nuclei (Loewenstein et al. 2001; Baganoff et al. 2003; Di Matteo et al. 2003; Ho et al. 2003). Tanaka \& Menou (2006) exploited these constraints to calculate mean free path of the observed gas. They suggested 
accretion in such systems are under weakly collisional condition. Moreover, they suggested thermal conduction as a possible mechanism by which the sufficient extra heating is provided in hot accretion flows. In following, Johnson \& Quataert (2007) studied the effects of electron thermal conduction on the properties of a spherical hot accretion flows. Their model is applicable for Sgr A* in the Galactic centre. Because, electron heat conduction is important for low accretion rate systems. They also found a supervirial temperature in the presence of thermal conduction. Similar to Tanaka \& Menou (2006), they assumed a steady state model, but they solved their equations numerically. Abbassi et al. (2008) presented a set of self-similar solutions for ADAFs with a toroidal magnetic field in which the saturated thermal conduction has a important role in the energy transport in the radial direction.

Since the observational evidences and magnetohydrodynamics (MHD) simulations have expressed the toroidal magnetic field and the magnetic diffusivity are important in accretion flows (see Faghei 2011a and references therein), Faghei (2011a) examined the selfsimilar solutions of viscous and resistive ADAFs in the presence of a toroidal magnetic field. But, he did not consider the effects of thermal conduction in his model. While, the recent studies of resistive accretion flows have represented that thermal conduction can play an importance role in such systems (e.g. Sharma et al. 2008; Ghanbari et al. 2009). Sharma et al. (2008) studied the effects of thermal conduction on magnetized spherical accretion flows using global axisymmetric $\mathrm{MHD}$ simulations. In their model, when the magnetic energy density becomes comparable to the gravitational potential energy density, the plasma due to resistivity is heated to roughly the virial temperature, the mean inflow becomes highly subsonic, and most of the energy released by accretion is transported to large radii by thermal conduction and the accretion rate became much smaller than Bondi accretion rate. Moreover, they found that for a larger values of conductive parameter, energy transport through thermal conduction becomes the dominant energy transport mechanism at small radii. Ghanbari et al. (2009) studied a two-dimensional advective accretion disc bathed in the poloidal magnetic field of a central accretor in the presence of thermal conduction. They did not consider toroidal component of magnetic field and for simplicity assumed the resistivity as a constant. They studied induction equation of magnetic field in a steady state that is not according to anti-dynamo theorem (e.g. Cowling 1981) and is useful only in particular systems where the magnetic dissipation time is much longer than the age of the system. On the other hand, this assumption implies that the flow is in balance between escape and creation of the magnetic field.

In this paper, we adopt the presented solutions by Tanaka \& Menou (2006) and Faghei (2011a). Thus, we will investigate the influences of thermal conduction on a viscous and resistive ADAF in the presence of a toroidal magnetic field. Moreover, it is assumed that magnetic diffusivity in the present model is not constant, and escaping and creating of magnetic field are unbalanced. From some aspects will be shown that the present model is in according with the observations and the resistive MHD simulations. The paper is organized as follows. In section 2, the basic equations of constructing a model for quasi-spherical magnetized advection dominated accretion flow with thermal conduction will be defined. In section 3, self- similar method for solving equations which govern the behaviour of the accreting gas was utilized. The summary of the model will appear in section 4 .

\section{Basic Equations}

We suppose a rotating and accreting gas around a Schwarzschild black hole of mass $M$. The flow is assumed to be in advection dominated stage, where viscous and resistive heating are balanced by the advection cooling and thermal conduction. We use a spherical coordinate $(r, \theta, \phi)$ centred on the accreting object. Furthermore, the flow is assumed to be steady and axisymmetric $\left(\partial_{t}=\partial_{\phi}=0\right)$, and the equations will be considered in the equatorial plane, $\theta=\pi / 2$. Thus, all flow variables are a function of only $r$. For the sake of simplicity, the general relativistic effects are ignored and Newtonian gravity is used. The magnetic field in the present model has only a toroidal component. Under assumptions, the model is described by the following equations:

The continuity equation with mass loss is

$\frac{1}{r^{2}} \frac{d}{d r}\left(r^{2} \rho v_{r}\right)=\dot{\rho}$,

where $\rho$ is density, $v_{r}$ is the radial infall velocity, and $\dot{\rho}$ the mass-loss rate per unit volume.

The radial equation of momentum is

$v_{r} \frac{d v_{r}}{d r}=r\left(\Omega^{2}-\Omega_{K}^{2}\right)-\frac{1}{\rho} \frac{d}{d r}\left(\rho c_{s}^{2}\right)-\frac{c_{A}^{2}}{r}-\frac{1}{2 \rho} \frac{d}{d r}\left(\rho c_{A}^{2}\right)$,

where $\Omega$ is the angular velocity of the flow, $\Omega_{K}=$ $\sqrt{G M / r^{3}}$ is the Keplerian angular velocity, $c_{s}$ the isothermal sound speed, which is defined as $c_{s}^{2}=p_{\text {gas }} / \rho$, $p_{\text {gas }}$ being the gas pressure, and $c_{A}$ is Alfven speed, which is defined as $c_{A}^{2}=B_{\varphi}^{2} / 4 \pi \rho=2 p_{\text {mag }} / \rho, p_{\text {mag }}$ 
being the magnetic pressure. The angular momentum transfer equation is

$\rho v_{r} \frac{d}{d r}\left(r^{2} \Omega\right)=\frac{1}{r^{2}} \frac{d}{d r}\left[\nu \rho r^{4} \frac{d \Omega}{\partial r}\right]$,

where the right-hand side of above equation describes the effects of viscous torques due to shear ( $\nu$, here, is kinematic coefficient of viscosity). As noted in the introduction, we assume both of the kinematic coefficient of viscosity and the magnetic diffusivity due to turbulence in the accretion flow. Thus, it is reasonable to use these parameters in analogy to the $\alpha$-prescription of Shakura \& Sunyaev (1973) for the turbulent,

$\nu=P_{m} \eta=\alpha \frac{c_{s}^{2}}{\Omega_{K}}$,

where $P_{m}$ is the magnetic Prandtl number, which is assumed a constant of order of unity, $\eta$ is the magnetic diffusivity, and $\alpha$ is a free parameter less than unity.

The energy equation becomes

$$
\begin{array}{r}
\frac{v_{r}}{\gamma-1} \frac{d}{d r}\left(\rho c_{s}^{2}\right)+\frac{\gamma}{\gamma-1} \frac{\rho c_{s}^{2}}{r^{2}} \frac{d}{d r}\left(r^{2} v_{r}\right)= \\
Q_{\text {diss }}-Q_{\text {rad }}+Q_{\text {cond }},
\end{array}
$$

where $Q_{\text {diss }}=Q_{v i s}+Q_{\text {resis }}$ is the dissipation rate by viscosity $Q_{v i s}$ and resistivity $Q_{\text {resis }}, Q_{\text {rad }}$ represents the energy loss through radiative cooling, and $Q_{\text {cond }}$ is the energy transported by thermal conduction. For the right-hand side of the energy equation, we can write

$Q_{a d v}=Q_{d i s s}-Q_{\text {rad }}+Q_{c o n d}$

where $Q_{a d v}$ is the advective transport of energy. We exploit the advection factor, $f=1-Q_{\text {rad }} / Q_{\text {diss }}$, that describes the fraction of the dissipation energy which is stored in the accretion flow and advected into the central object rather than being radiated away. In general, the advection factor depends on the details of the heating and radiative cooling mechanisms and will vary by position (e.g. Watari 2006, 2007; Sinha et al. 2009). Here, we assume a constant $f$ for simplicity. Clearly, the flow in the case of $f=1$ is in the extreme limit of no radiative cooling and in the limit of efficient radiative cooling, we have $f=0$.

As mentioned, the inner regions of hot accretion flows are collisionless and the electron mean free path due to Coulomb collision is larger than the radius. This property is described as saturation (Cowie \& McKee 1977). The traditional equation of heat flux due to thermal conduction, $F_{\text {cond }}=-\kappa \nabla T$ which $\kappa$ being the thermal conduction coefficient, is not valid for such systems. Because this equation is suitable for the collisional plasma, in which mean free path of electron energy exchange is smaller than temperature scale height.
Cowie \& McKee (1977) derived the heat flux for the collisionless plasma as

$F_{\text {sat }}=5 \phi_{s} \rho c_{s}^{3}=5 \phi_{s} p \sqrt{\frac{p}{\rho}}$,

where $\phi_{s}$ is a factor is less than unity and is called as saturation constant. Now, the viscous and resistive heating rates and the energy transport by thermal conduction are expressed as

$Q_{v i s}=\nu \rho r^{2}\left(\frac{\partial \Omega}{\partial r}\right)^{2}$

$Q_{\text {resis }}=\frac{\eta}{4 \pi} \mathbf{J}^{2}$

$Q_{\text {cond }}=-\left|\frac{1}{r^{2}} \frac{\partial}{\partial r}\left(r^{2} F_{\text {sat }}\right)\right|$

where $\mathbf{J}=\nabla \times \mathbf{B}$ is the current density, $\mathbf{B}$ being the magnetic field. We used a minus sign in equation (10). Because we want thermal conduction as energy transport mechanism outward. On the other hand, heat conduction flux will be behaved like a cooling mechanism in accretion flow. Finally, since we consider only the toroidal field, the induction equation with field escape can be written as

$\frac{1}{r} \frac{d}{d r}\left[r v_{r} B_{\varphi}-\eta \frac{d}{d r}\left(r B_{\varphi}\right)\right]=\dot{B}_{\varphi}$.

where $B_{\varphi}$ is the toroidal component of magnetic field and $\dot{B}_{\varphi}$ is the field escaping/creating rate due to a magnetic instability or dynamo effect. This induction equation is rewritten as

$$
\frac{1}{r} \frac{d}{d r}\left[\sqrt{4 \pi \rho c_{A}^{2}}\left(r v_{r}-\frac{\alpha}{4 \beta P_{m}} \frac{1}{r \rho \Omega_{K}} \frac{d}{d r}\left(r^{2} \rho c_{A}^{2}\right)\right)\right]=
$$

where $\beta$ is the degree of magnetic pressure to the gas pressure and can be defined by

$\beta=\frac{p_{\text {mag }}}{p_{\text {gas }}}=\frac{1}{2}\left(\frac{c_{A}}{c_{s}}\right)^{2}$.

In this paper, we apply the steady self-similar methods to solve the system equations. Thus, this parameter will be constant throughout the disc. While, Khesali \& Faghei $(2008,2009)$ showed that it varies by position. In hot accretion flows, typical value of $\beta$ is in the range 0.01-1 (e.g. De Villiers et al. 2003; Beckwith et al. 2008). Here, we will also consider the magnetically dominated case $(\beta>1)$. Because, when thermal instability happens in an ADAF, the MHD numerical 
simulations imply that the thermal pressure rapidly decreases while the magnetic pressure increases due to the conservation of magnetic flux (Machida et al. 2006). This will result in large $\beta$ and forms a magnetically dominated accretion flow (Bu et al. 2009).

\section{Self-Similar Solutions}

\subsection{Analysis}

The self-similar method is useful to understand of the physics of accretion flows. This method is familiar due to its wide range of applications in many research fields of astrophysics. Self-similar solution, although constituting only a limited part of problem, is often useful to understand the basic behaviour of the system. Thus, in order to seek similarity solutions for the above equations, we seek solutions in the following form:

$v_{r}(r)=-c_{1} \alpha \sqrt{\frac{G M_{*}}{r}}$
$\Omega(r)=c_{2} \sqrt{\frac{G M_{*}}{r^{3}}}$
$c_{s}^{2}(r)=c_{3} \frac{G M_{*}}{r}$
$c_{A}^{2}(r)=\frac{B_{\varphi}^{2}}{4 \pi \rho}=2 \beta c_{3} \frac{G M_{*}}{r}$

where coefficients $c_{1}, c_{2}$, and $c_{3}$ are determined later. We assume a power-law relation for density

$\rho(r)=\rho_{0} r^{s}$

where $\rho_{0}$ and $s$ are constant. By using above selfsimilar quantities, the mass-loss rate and the field escaping/creating rate must have the following form:

$\dot{\rho}(r)=\dot{\rho}_{0} r^{s-3 / 2}$

$\dot{B}_{\varphi}(r)=\dot{B}_{0} r^{\frac{s-4}{2}}$

where $\dot{\rho}_{0}$ and $\dot{B}_{0}$ are constant.

Substituting the above solutions in the continuity, momentum, angular momentum, energy, and induction equations $[(1)-(3),(5)$, and (11)], we can obtain the following relations:

$\dot{\rho}_{0}=-\left(s+\frac{3}{2}\right) \alpha \rho_{0} c_{1} \sqrt{G M_{*}}$,

$-\frac{1}{2} c_{1}^{2} \alpha^{2}=c_{2}^{2}-1-c_{3}[s-1+\beta(1+s)]$

$c_{1}=3(s+2) c_{3}$

$$
\begin{array}{r}
-\alpha c_{1}\left[\frac{2(s-1)+3 \gamma}{\gamma-1}\right]=\alpha f\left[\frac{9}{2} c_{2}^{2}+\frac{\beta}{P_{m}} c_{3}(1+s)^{2}\right] \\
-10 \phi_{s} c_{3}^{1 / 2}\left|s+\frac{1}{2}\right|
\end{array}
$$

$\dot{B}_{0}=-\frac{\alpha s}{2} G M_{*} \sqrt{2 \pi \rho_{0} \beta c_{3}}\left[2 c_{1}+\frac{c_{3}}{P_{m}}(1+s)\right]$.

Above equations express for $s=-3 / 2$, there is no mass loss, while for $s>-3 / 2$ mass loss (wind) exists. After algebraic manipulations, we obtain an algebraic equation for $c_{3}$ :

$$
\begin{array}{r}
\frac{81}{8} \alpha^{3}(s+2)^{2} f c_{3}{ }^{2}+c_{3}\left[\frac{3 \alpha(s+2)}{2} \times \frac{2(s-1)+3 \gamma}{\gamma-1}\right. \\
\left.-\frac{9 \alpha f}{4}\left(\frac{2 \beta}{9 P_{m}}(s+1)^{2}+(s+1) \beta+s-1\right)\right] \\
-5 \phi_{s}\left|s+\frac{1}{2}\right| \sqrt{c_{3}}-\frac{9}{4} f \alpha=0
\end{array}
$$

and the rest of the physical variables are

$$
\begin{aligned}
& \dot{\rho}_{0}=-3 \alpha \rho_{0} \sqrt{G M_{*}}(s+2)\left(s+\frac{3}{2}\right) c_{3}, \\
& c_{1}=3 c_{3}(s+2), \\
& c_{2}^{2}=1-\frac{9 \alpha^{2}}{2}(s+2)^{2} c_{3}^{2}+c_{3}[(s+1) \beta+s-1], \\
& \dot{B}_{0}=-3 \alpha s G M_{*}(s+2)^{3 / 2} c_{3}^{3 / 2} \sqrt{6 \pi \rho_{0} \beta} \times \\
& {\left[1+\frac{s+1}{6 P_{m}(s+2)}\right] .}
\end{aligned}
$$

We can solve algebraic equation (26) numerically and clearly only real roots which correspond to positive $c_{1}$ 
5
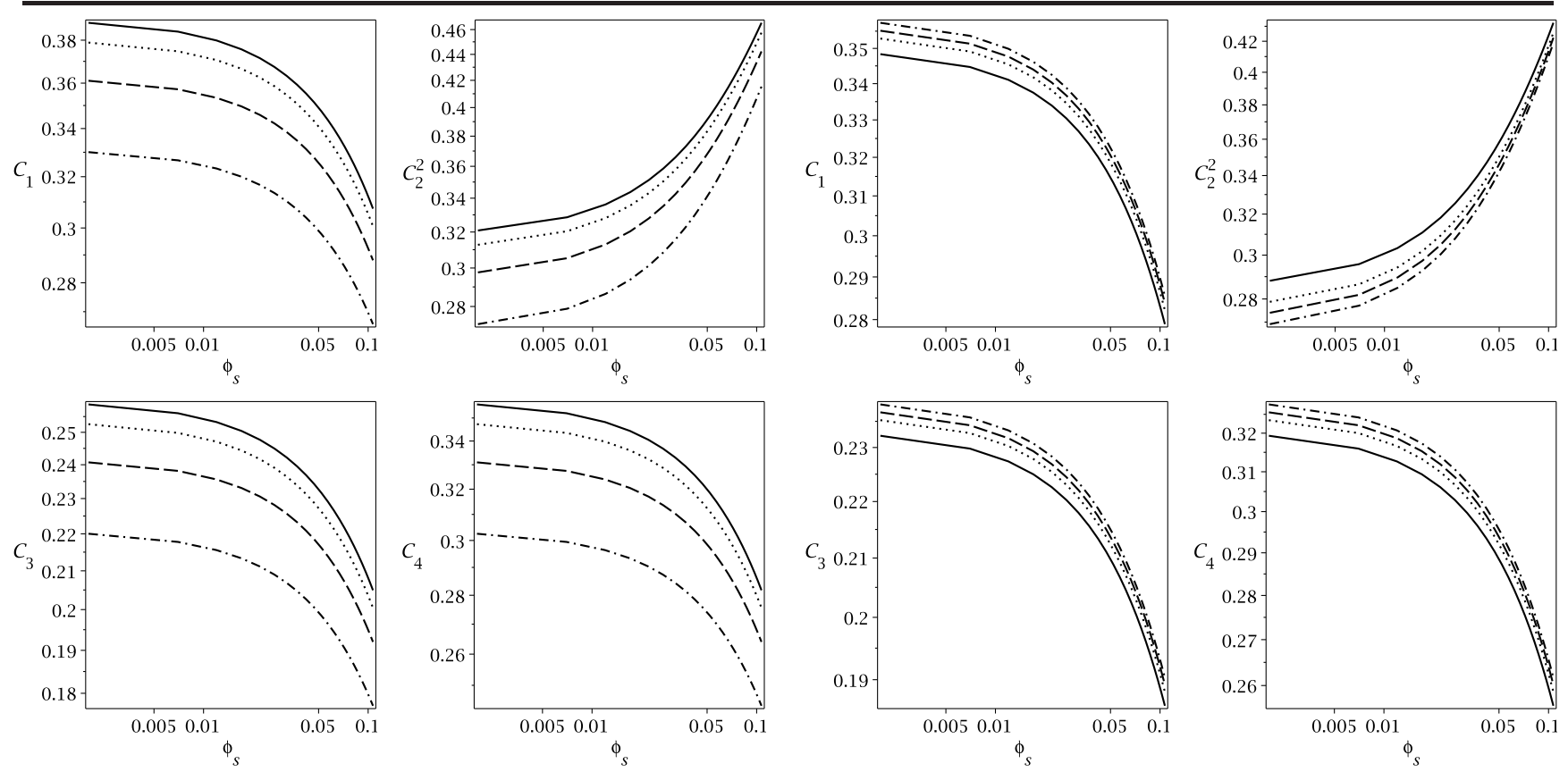

Fig. 1 Physical quantities of the flow as a function of saturation constant for $\gamma=1.3, \alpha=0.5, f=1$, and $P_{m}=100$. Solid, dotted, dashed, and dot-dashed lines represent $\beta=0.1,0.3,0.7$, and 1.5 .

are physically acceptable. Without thermal conduction, i.e. $\phi_{s}=0,(26)$ and similarity solutions reduce to Faghei (2011a). But our main algebraic equation includes thermal conduction.

\subsection{Numerical Results}

Now, we consider the behaviour of solutions in the presence of thermal conduction. But in this paper, only case of no wind $(s=-3 / 2)$ is considered that $\dot{\rho}_{0}=0$ and $\dot{B}_{\varphi} \propto r^{-11 / 4}$. In addition to introduced coefficients, we also define a new parameter of $c_{4}$ that is the right-hand side of equation (24)

$c_{4}=\alpha f\left[\frac{9}{2} c_{2}^{2}+\frac{\beta}{P_{m}} c_{3}(1+s)^{2}\right]-10 \phi_{s} c_{3}^{1 / 2}\left|s+\frac{1}{2}\right|$.

Equations (5), (24), and (31) imply that the parameter $c_{4}$ is the advection transport of energy. The behaviour of coefficients $c_{i}$ as a function of $\phi_{s}$ are shown in Figures 1-3. Moreover, Figure 1 represents the profiles of physical quantities for several values of the magnetic pressure fraction, i. e. $\beta=0.1,0.3,0.7$, and 1.5 . The value of $\beta$ measures the strength of magnetic field, and a larger $\beta$ denotes a stronger magnetic field. Figure 2 represents the profiles of physical quantities for several values of

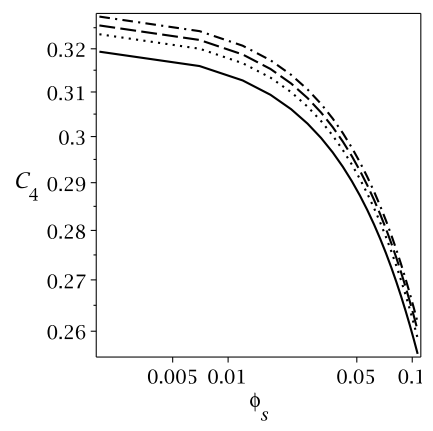

Fig. 2 Same as Fig. 1, but $\beta=1.0$, and solid, dotted, dashed, and dot-dashed lines represent $P_{m}=\infty, 1,2 / 3$, and $1 / 2$.

magnetic Prandtl number, i. e. $P_{m}=\infty, 1,2 / 3$, and $1 / 2$. The smaller values of $P_{m}$ denotes a stronger magnetic diffusivity, $\eta$. Figure 3 shows the profiles of physical quantities for several values of adiabatic index, $i$. e. $\gamma=1.2,1.25,1.3$, and 1.35 .

The solutions in Figures 1-3 imply that the radial infall velocity, $c_{1}$, and the sound speed, $c_{3}$, both decrease with the magnitude of conduction, while the squared angular velocity, $c_{2}^{2}$, increases. These properties are qualitatively consistent with dynamical analysis of Faghei (2011b). One and two dimensional simulations of hot accretion flows have also shown that thermal conduction reduces the flow temperature (Sharmal et al. 2008; Wu et al. 2010). The profiles of advection transport of energy, $c_{4}$, in Figures 1-3 imply that thermal conduction behaves as a cooling mechanism, resulting in a local decrease of the gas temperature relative to the original ADAF solution. At the same time, the gas adjust its angular velocity (which increases the level of viscous dissipation) and reduces its inflow speed.

Figure 1 shows the radial velocity, sound speed, and advection transport of energy decrease by adding the magnetic pressure fraction, $\beta$. These properties are qualitatively consistent with results of $\mathrm{Bu}$ et al. (2009) and Faghei (2011a). Moreover, the angular velocity decreases with the magnitude of magnetic field that is in according with results of Khesali \& Faghei (2009) and Faghei (2011a). 

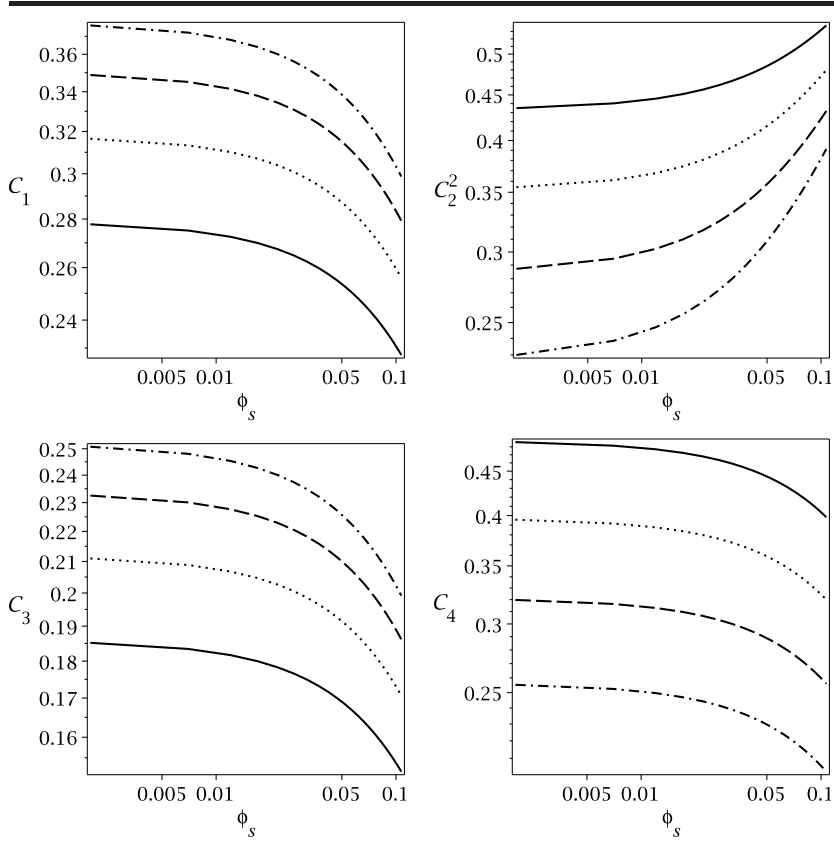

Fig. 3 Same as Fig. 1, but $\beta=1.0$, and solid, dotted, dashed, and dot-dashed lines represent $\gamma=1.2,1.25,1.3$, and 1.35 .

Figure 2 shows the magnetic diffusivity has the opposite effects of thermal conduction on the physical variables. As, by adding the magnetic diffusivity, $P_{m}^{-1}$, the radial velocity, sound speed, and advection transport of energy increase, while the rotational velocity decrease. These results are similar to resistive ADAF models without thermal conduction (e. g. Faghei 2011a).

Figure 3 represents the gas adiabatic index similar to magnetic diffusivity has the opposite effects of thermal conduction on the physical quantities. As, with the magnitude of $\gamma$, the inflow and sound speed increase, while the rotational velocity decreases. This is in accord with dynamical study of hot accretion flow (Faghei 2011b). Moreover, Figure 3 shows that the gas adiabatic index contributes with thermal conduction to reduce advection transport of energy. It can be due to decrease of rotational velocity by adding $\gamma$, which reduces the level of viscous dissipation.

The studies of hot accretion flows (e. g. Shadmehri 2008) imply that the solution for a given set of the input parameters reaches to a non-rotating limit at a specific of $\phi_{s}$ which we denote it by $\phi_{s}^{c}$. With zero insertion of $c_{2}$ in equations (22)-(24), $\phi_{s}^{c}$ can be written as

$$
\sqrt{\phi_{s}^{c}=\frac{1}{60}\left[\frac{\beta f}{P_{m}}-\frac{5 / 3-\gamma}{\gamma-1}\right] \times} \sqrt{2(\beta+5)\left[-1+\sqrt{1+\frac{18 \alpha^{2}}{(\beta+5)^{2}}}\right]} .
$$

We can not extend the studies beyond $\phi_{s}^{c}$, because the right-hand side of equation (29) becomes negative and a negative $c_{2}^{2}$ is clearly unphysical. As, the rotational velocity profiles in Figures 1-3 show, we have selected the input parameters that $c_{2}^{2}$ is positive. The behaviour of critical saturation constant, $\phi_{s}^{c}$, as a function of $\beta$ for several values of magnetic Prandtl number is shown in Figures 4. In left panel of Figure 4, the viscosity parameter value is $\alpha=0.1$, and in right panel is $\alpha=0.2$. The profiles of Figure 4 show the critical saturation constant highly depends on magnetic pressure fraction, $\beta$, magnetic Prandtl number, $P_{m}$, and viscosity parameter, $\alpha$. As, higher values of $\beta$ corresponds to larger $\phi_{s}^{c}$. Critical saturation constant also increases with higher value of $\alpha$. Since, magnetic diffusivity is proportional to $P_{m}^{-1}$, Figure 4 shows that the magnetic diffusivity similar to magnetic field increases $\phi_{s}^{c}$ value.

As mentioned in the introduction, Sharma et al. (2008) by resistive MHD simulation studied a spherical accretion with thermal conduction. They found for even modest thermal conductivities, conduction is the significant mechanism of energy. Here, to compare conduction mechanism to others, we study the ratio of energy transport by thermal conduction, $Q_{\text {cond }}$, to the gas heating rate by viscosity $Q_{v i s}$ and resistivity $Q_{\text {resis. }}$. Such solutions are shown in Figure 5 for two cases of non-resistive (left-panel) and resistive (rightpanel) flows. The solutions imply that thermal conduction is the significant energy mechanism in the flow. This result confirms simulation of Sharma et al. (2008). Moreover, the ratio of $Q_{\text {cond }}$ to $Q_{\text {diss }}$ increases slightly by adding the magnetic field and does not change for different values of magnetic Prandtl number. Because, a large fraction of $Q_{\text {diss }}$ is generated by viscous dissipation.

\section{Summary and Discussion}

The collision timescale between ions and electrons in hot accretion flows is longer than the inflow timescale. Thus, the inflow plasma is collisionless, and transfer of energy by thermal conduction can be dynamically important. The low collisional rate of the gas is confirmed by direct observation, particularly in the case of the Galactic centre (Quataert 2004; Tanaka \& Menou 2006) and in the intracluster medium of galaxy clusters (Sarazin 1986).

In this paper, the structure of a magnetized ADAF in the presence of resistivity and thermal conduction is investigated. We assumed the magnetic field has a purely toroidal component. We adopted the presented solutions by Tanaka \& Menou (2006) and Faghei (2011a). 

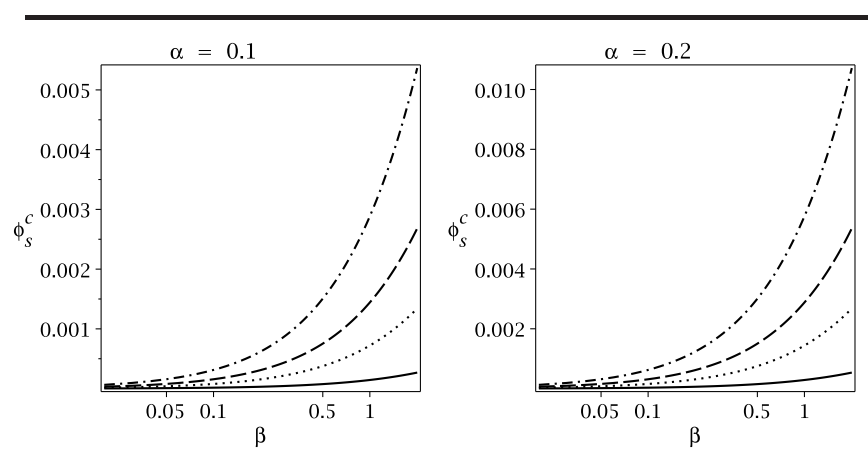

Fig. 4 The critical saturation constant as a function of the ratio of magnetic pressure to gas pressure. Solid, dotted, dashed, and dot-dashed lines represent $P_{m}=10,5,1$, and 0.5. The input parameters are set to $\gamma=5 / 3, f=1$, $s=-3 / 2$, and the viscous parameter $\alpha$ in left-panel is 0.1 and in right-panel is 0.2 .

Thus, we assumed that angular momentum transport is due to viscous turbulence and the $\alpha$ - prescription is used for the kinematic coefficient of viscosity. We also assumed the flow does not have a good cooling efficiency and so a fraction of energy accretes along with matter on to the central object. In order to solve the equations that govern the structure behaviour of magnetized ADAF with thermal conduction, we have used steady self-similar solution.

The solutions showed the radial infall velocity and sound speed in the presence of thermal conduction both decrease, while angular velocity increase. These properties are consistent with dynamical study of hot accretion flow (e. g. Faghei 2011b) and from some aspects also are in accord with simulations of Sharma et al. (2008) and Wu et al. (2010). Moreover, the solutions represent the magnetic diffusivity and thermal conduction have the opposite effects on physical quantities. For a moderate thermal conduction, the solutions imply that thermal conduction can play an important role in energy mechanism of the system. This property is qualitatively consistent with non-ideal simulations of Sharma et al. (2008).

In the present model, accretion flow is studied in one-dimensional approach and ignored from latitudinal dependence of physical quantities. There are some researches in two-dimensional approach that express the importance of such studies (Tanaka \& Menou 2006; Ghanbari et al. 2009; Wu et al. 2010 ). Thus, latitudinal study of present model can be investigated in other research. Here, we used a saturated heat conduction flux. While, there are some studies with unsaturated heat flux (e. g. Shcherbakkov \& baganoff 2010) that show a good agreement with observations. Thus, the
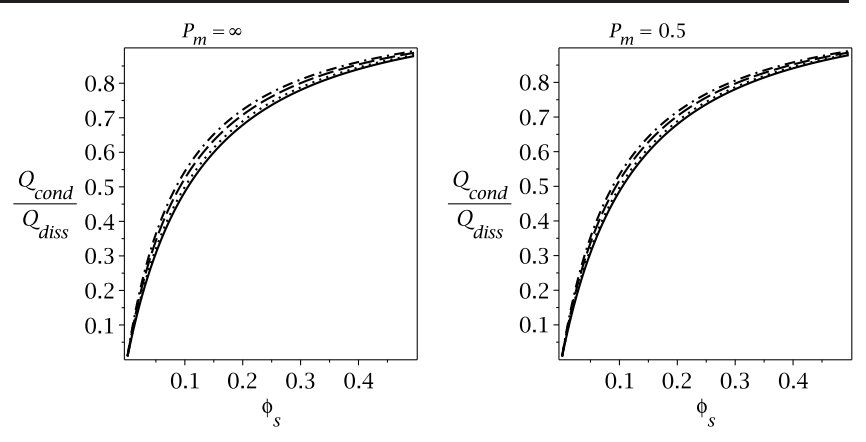

Fig. 5 The ratio of energy transport by thermal conduction, $Q_{\text {cond }}$, to the gas heating rate by viscosity $Q_{v i s}$ and resistivity $Q_{\text {resis }}$ as a function of saturation constant. Solid, dotted, dashed, and dot-dashed lines represent $\beta=$ $0.0,1.0,3.0$, and 5.0. The input parameters are set to $\gamma=4 / 3, f=1, s=-3 / 2, \alpha=0.5$, and the magnetic Prandtl number in left-panel is $\infty$ and in right-panel is 0.5 .

study of the present model in a unsaturated case will be interesting.

\section{Acknowledgements}

I wish to thank the anonymous referee for very useful comments that helped me to improve the initial version of the paper. I would also like to thank Roman V. Shcherbakov for his helpful comments.

\section{References}

Abbassi S., Ghanbari G., Najjar S., 2008, MNRAS, 388, 663 Abramowicz, M., Chen, X., Kato, S., Lasota, J. P., Regev, O., 1995, ApJ, 438, L37

Baganoff, F. K., et al. 2003, ApJ, 591, 891

Blandford, R. D., Begelman, M. C. 1999, MNRAS, 303, L1

Bu D., Yuan F., Xie F., 2009, MNRAS, 392, 325

Cowie L. L., McKee C. F., 1977, ApJ, 211, 135

Cowling, T. G., 1981, ARA\&A, 19, 115

Di Matteo T., Allen S. W., Fabian A. C., Wilson A. S., Young, A. J. 2003, ApJ, 582, 133

Di Matteo T., Quataert, E., Allen S. W., Narayan R., Fabian A. C., 2000, MNRAS, 311, 507

Esin A. A., McClintock J.E., Narayan R. 1997, ApJ, 489, 865

Esin A. A., McClintock J. E., Drake J. J., Garcia M. R., Haswell C. A., Hynes R. I., Muno M. P., 2001, ApJ, 555, 483

Faghei, K., 2011a, J. Astrophys. Astr., accepted

Faghei, K., 2011b, MNRAS, accepted

Ghanbari J., Abbassi S., Ghasemnezhad M., 2009, MNRAS, 400, 422

Ho L. C., Terashima Y., Ulvestad J. S., 2003, ApJ, 589, 783 
Ichimaru, S. 1977, ApJ, 214, 840

Johnson B. M., Quataert E., 2007, ApJ, 660, 1273

Khesali, A., Faghei, K., 2008, MNRAS, 389, 1218

Khesali, A., Faghei, K., 2009, MNRAS, 398, 1361

Lasota J.-P., Abramowicz M. A., Chen X., Krolik J., Narayan R., Yi I., 1996, ApJ, 462, 142L

Loewenstein M., Mushotzky R. F., Angelini L., Arnaud K. A., Quataert, E., 2001, ApJ, 555, L21

Machida M., Nakamura K. E., Matsumoto R., 2006, PASJ, 58, 193

Melia F., Falcke H. 2001, ARA\&A, 39, 309

Menou K., Esin, A. A., Narayan R., Garcia M. R., Lasota J.-P., McClintock J. E., 1999, ApJ, 520, 276

Narayan R. 2002, in Lighthouses of the Universe, ed. Gilfanov M., Sunyaev R. A., Churazov E. ( Berlin: Springer), 405

Narayan R., Mahadevan R., Grindlay J. E., Popham R. G., Gammie C., 1998a, ApJ, 492, 554

Narayan, R., Mahadevan, R., Quataert, E. 1998b, in Theory of Black Hole Accretion Disks, ed. Abramowicz M., Bjornsson G., Pringle J. (Cambridge: Cambridge Univ. Press), 148

Narayan R., Quataert E., 2005, Science, 307, 77

Narayan, R., Yi, I., 1994, ApJ, 428, L13

Ogilvie, G. I., 1999, MNRAS, 306, L9O

Quataert E., 2004, ApJ, 613, 322

Rees, M. J., Begelman, M. C., Blandford, R. D., Phinney, E. S. 1982, Nature, 295, 17

Sarazin C. L., 1986, Rev. Mod. Phys., 58, 1

Shadmehri, M., 2008, Ap\&SS, 317, 201

Shakura N. I., Sunyaev R. A., 1973, A\&A, 24, 337

Sharma P., Quataert E., Stone J. M., 2008, MNRAS, 389, 1815

Shcherbakov R. V., Baganoff F. K., 2010, ApJ, 716, 504

Sinha M., Rajesh S. R., Mukhopadhyay B., 2009, RAA, 9, 1331

Tanaka T., Menou K., 2006, ApJ, 649, 345

Watarai, K. 2006, ApJ, 648, 523

Watarai, K. Y. 2007, PASJ, 59, 443

Wu M., Yuan F., Bu D., 2010, ScChG, 53S, 168

This manuscript was prepared with the AAS LATEX macros v5.2. 